\title{
Irregular Repetition and Single Parity Check Coded BICM-ID Using Extended Mapping -Optimal Node Degree Allocation-
}

\author{
Kisho Fukawa*, Dan Zhao*, Antti Tolli ${ }^{\dagger}$, \\ Tad Matsumoto* ${ }^{* \dagger}$ (Invited) \\ *Japan Advanced Institute of Science and Technology(JAIST), Japan \\ Email: \{matumoto, k.fukawa, dan.zhao\}@jaist.ac.jp \\ and \\ ${ }^{\dagger}$ Center for Wireless Communication at University of Oulu,Finland \\ Email: \{tadashi.matsumoto, antti.tolli\}@ee.oulu.fi
}

\begin{abstract}
Since BICM-ID is a concatenation between channel coding and mapping, its performances strongly depend on the matching between mapping rule and code structure. In our previous papers, we proposed a yet very simple, close Shannonlimit achieving BICM-ID system. It uses very simple codes, irregular repetition and single parity check codes, combined with extended mapping. Even though we know that the key role played towards the optimal design of the proposed code is the degree allocation for variable nodes, the irregular degree allocation to the node degrees were determined only empirically, by try-anderror. This paper shows that the problem of the optimal degree allocation for the proposed BICM-ID technique can be solved by using linear programming technique. Results shows we can achieve better matching between the de-mapper and decoder curves, by which we can achieve even closer threshold to the Shannon limit and also lower error floor.
\end{abstract}

\section{INTRODUCTION}

Bit-Interleaved Coded Modulation with Iterative Detection/Decoding (BICM-ID) [1] has been recognized as being a bandwidth efficient coded modulation and transmission scheme, of which transmitter is comprised of a concatenation of encoder and bit-to-symbol mapper separated by a bit interleaver. Iterative detection-and-decoding takes place at the receiver, where extrinsic log likelihood ratio (LLR) obtained as the result of the maximum a posteriori probability (MAP) algorithm for demapping/decoding is forwarded to the decoder/demapper via de-interleaver/interleaver, and used as the a priori LLR for decoding/demapping, according to the standard turbo principle.

Performances of BICM-ID have to be evaluated by the convergence and asymptotic properties [2], which are represented by the threshold signal-to-noise power ratio (SNR) and bit error rate (BER) floor, respectively. In principle, since BICM-ID is a serially concatenated system, analyzing its performances can rely on the area property [3] of the extrinsic information transfer (EXIT) chart. Therefore, the transmission link design based on BICM-ID falls into the

This work was supported by the Japanese government funding program, Grant-in-Aid for Scientific Research (B), No. 20360168 issue of matching between the demapper and decoder EXIT curves. Various efforts have been made in the seek of better matching between the two curves for minimizing the gap while still keeping the tunnel open, aiming, without requiring heavy detection/decoding complexity, at achieving lower threshold SNR and BER floor. Reference [4] introduces a technique that makes good matching between the detector and decoder EXIT curves using low density parity check (LDPC) code in multiple input multiple output (MIMO) spatial multiplexing systems.

It has been believed that a combination of Gray mapping and Turbo or LDPC codes achieves the best performance comparing with other combinations for BICM-ID. However, Ref. [5] proposes another approach towards achieving good matching between the two curves by introducing different mapping rules, such as anti-Gray mapping, which allows the use of even simpler codes, such as convolutional codes, and with which still it is possible to achieve BER pinch-off (corresponding to the threshold SNR). Another good idea that can provide us with design flexibility is extended mapping (EM) presented by [6], [7] where with $2^{m}$-QAM, more than $m$ bits, $l_{\text {map }}$, are allocated to one signal point in the constellation. With this mapping rule, the left-most point of the demapper EXIT function has a lower value than with the Gray mapping, but the right-most point becomes higher. With this setting, the demapper EXIT function achieves better matching with even weaker codes such as small memory length convolution codes, and of the most practical importance is the fact that hardware complexity of the modulator and demodulator remains the same as that with the original $2^{m}$-QAM.

In our previous publications [8], [9], we proposed a yet very simple, close Shannon-limit achieving BICM-ID system. It uses very simple codes, irregular repetition and single parity check codes, combined with extended mapping. Even though we know that the key role played towards the optimal design of the proposed code is the degree allocation for variable nodes, in [8], the irregular degree allocation to the node degrees were determined only empirically, by try-and-error. The major contribution of this paper is to show that the problem of the 
optimal degree allocation for the proposed BICM-ID technique can be solved by using linear programming technique. Results shows we can achieve better matching between the de-mapper and decoder curves, by which we can achiever even closer threshold to the Shannon limit and also lower error floor.

The paper is organized as follows. The system model is described in Section II. Since the code structures of our proposed BICM-ID system were provided in our previous publications, only basic structure is introduced in Section II. The convergence property of the proposed technique was also analyzed in the previous publication, and therefore, only the results are shown in this paper. The major contribution of this paper is provided in Section III where it is shown that the optimal degree allocation falls into a convex optimization, and furthermore, it can be solved by using linear programming technique. In Section IV chain simulation results as well as performance results are shown. Finally Section V concludes this paper.

\section{SYSTEM MODEL}

A schematic diagram of the proposed BICM-ID system is shown in Fig. 1.

\section{A. Transmitter}

The binary bit information sequence $\underline{u}$ to be transmitted is encoded by, first, a single parity check code where a single parity bit is added to every $d_{c}-1$ information bits, followed by a repetition code. $d_{c}$ is referred to as check node degree.



Fig. 1. System Model

The repetition times $d_{v}$, referred to as variable node degree, take different values in one whole block (=transmission frame); if $d_{v}$ takes several different values in a block, such code is referred to as having irregular degree allocation. It is assumed that throughout the paper $d_{c}$ takes only one identical value as in [4].

The coded bit sequence is bit-interleaved, segmented into $l_{m a p}$-bit segments, and then the each segment is mapped on to one of the $2^{m}$ constellation points for modulation. Since $l_{\text {map }}>m$ with the EM technique, more than one labels having different bit patterns in the segment are mapped on to each constellation point. However, there are many possible combinations of bit patterns to be allocated to the constellation points. This paper uses the labels assigned to the each constellation point, obtained by [6], so that, with full a priori information, the mutual information (MI) between the coded bit and the demapper output extrinsic LLR at the right most point of the demapper EXIT curve is maximized. Fig. 2 shows the optimal labeling for 4-QAM with $l_{m a p}=3$ and 5 . The complex-valued signal modulated according to the mapping rule, referred to as $\psi$, is finally transmitted to the wireless channel.

\section{B. Channel}

This paper assumes frequency flat block fading additive white Gaussian noise (AWGN) channel. If the channel exhibits frequency selectivity due to the multipath propagation, the receiver needs an equalizer to eliminate the inter-symbol interference. However, combining the technique presented in this paper with the turbo equalization framework [10]- [11] is rather straightforward.

It is assumed that transmission chain is properly normalized so that the received symbol energy-to-noise power spectral density ratio $E_{s} / N_{0}=1 / \sigma_{n}^{2}$; with this normalization, we can properly delete the channel complex gain term from the mathematical expression of the channel. The discrete time description of the received signal $y(k)$ is then expressed by

$$
y(k)=x(k)+n(k),
$$

where, with $k$ being the symbol timing index, $x(k)$ is the transmitted modulated signal with unit power, and $n(k)$ the zero mean complex AWGN component with variance $\sigma_{n}^{2}$ (i.e., $<|x(k)|^{2}>=1,<n(k)>=0$, and $\left\langle|n(k)|^{2}>=\sigma_{n}^{2}\right)$.

\section{Receiver}

1) De-mapper: At the receiver side, the iterative processing is invoked, where extrinsic information is exchanged between the demapper and decoder. The extrinsic LLRs calculated by soft input soft output (SISO) decoding/demapping are fed back and used for demapping/decoding as a priori LLR; the iteration continues until no more relevant gains in extrinsic MI can be achieved [12]; when such convergence point is reached, binary decisions are made on the information bits based on the a posteriori LLR at the variable node. Therefore, the larger the MI at the convergence point, the lower the BER floor, which is depending on the matching between the encoder and the mapping rule.

The demapper calculates from the received signal point $y(k)$, corrupted by AWGN, the extrinsic LLR of the $\mu^{t h}$ bit in the symbol transmitted at the $k^{\text {th }}$ symbol timing, by

$$
\begin{aligned}
& L_{e}\left[b_{\mu}(k)\right]= \\
& \ln \frac{\sum_{s \in S_{0}} \exp \left\{-\frac{|y-s|^{2}}{\sigma_{N}^{2}}\right\} \prod_{v=1, v \neq \mu}^{l_{\text {map }}} \exp \left(-b_{v}(s) L_{a}\left(b_{v}(s)\right)\right.}{\sum_{s \in S_{1}} \exp \left\{-\frac{|y-s|^{2}}{\sigma_{N}^{2}}\right\} \prod_{v=1, v \neq \mu}^{l_{\text {map }}} \exp \left(-b_{v}(s) L_{a}\left(b_{v}(s)\right.\right.}
\end{aligned}
$$

where $s$ denotes a signal point in the constellation, $S_{0}\left(S_{1}\right)$ indicates the set of the labels having the $\mu^{\text {th }}$ bit being $0(1)$, and $L_{a}\left(b_{v}(s)\right)$ the a priori LLR fed back from the decoder 


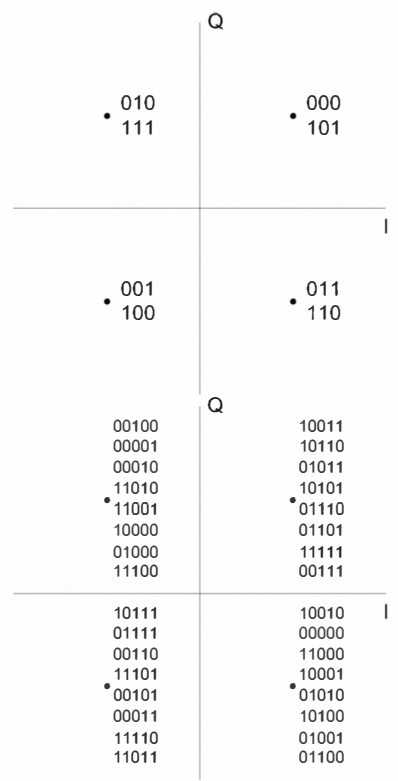

Fig. 2. Extended mapping 4-QAM $l_{m a p}=3$ and $l_{m a p}=5$, optimized with a priori information.

corresponding to the $v^{\text {th }}$ position in the label allocated to the signal point $s$.

2) Decoder: Fig. 3 shows a block diagram of the decoder with the structure. Decoding takes place segment-wise where, because of the irregular code structure, the variable node degrees $d_{v, i}$ have different values segment-by-segment.

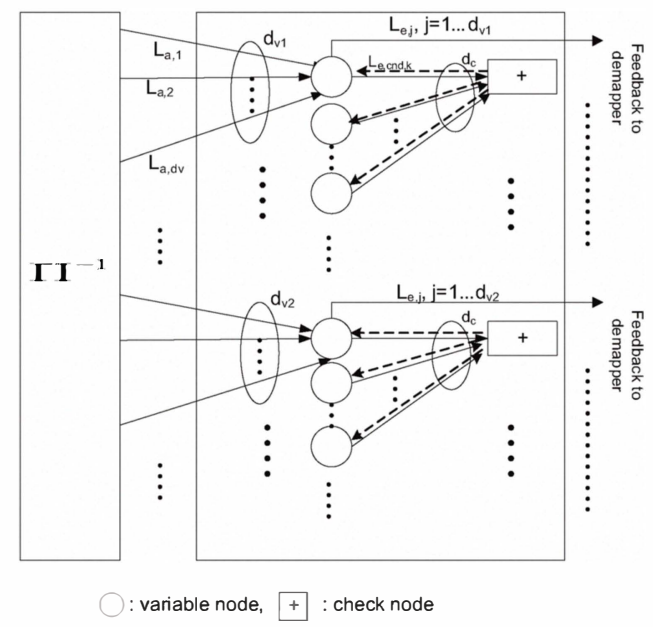

Fig. 3. Decoder Structure

The $d_{v, i}$ bits constituting one segment, output from the deinterleaver, are connected to a variable node, and $d_{c}$ variable nodes are further segmented and connected to a check node decoder; those demapper output bits in one segment, connected to the same variable node decoder, are not overlapping with other segments, and so is the case of the variable node segmentation. Therefore, no iterations in the decoder are required. The extrinsic LLR update for a bit at the check node is exactly the same as the check node operation in the LDPC codes, as

$$
L_{e, c n d, i}=\sum_{u=1}^{d_{c}} L_{a, c n d, u}
$$

where $\Psi \boxplus$ indicates the box-sum operator [13], and $L_{a, c n d, u}$ is the a priori LLR for the $u$-th bit in the $i$-th segment.

The extrinsic LLR, calculated by the check node decoder, is fed back to its connected variable node decoder, where it is further combined with $\left(d_{v, i-1}\right)$ a priori LLRs forwarded from the demapper via the de-interleaver, as

$$
L_{e, j}=L_{e, c n d, i}+\sum_{u=1, u \neq j}^{d_{v, i}} L_{a, u}
$$

This process is performed for the other bits in the same segment, and also for all the other segments independently in the same transmission block. Finally, the updated extrinsic LLRs obtained at the each variable node are interleaved, and fed back to the demapper.

The rate of the code is $\left(d_{c}-1\right) /\left(d_{c} \cdot \sum a_{i} \cdot d_{v, i}\right)$, and the spectrum efficiency is $l_{\text {map }} \cdot\left(d_{c}-1\right) /\left(d_{c} \cdot \sum a_{i} \cdot d_{v, i}\right)$ bits per symbol, where $a_{i}$ represents the ratio of variable nodes having degree $d_{v, i}$ in a block.

3) EXIT Functions: The distribution of LLRs of the coded transmitted bits at the output of the de-mapper, according to Eq. (2) was evaluated when calculating the EXIT function of the de-mapper. Fig. 4 shows EXIT curves for 4-QAM demappers with $l_{m a p}$ as a parameter, where the labels were determined so that the EXIT curve has the largest decay. Also, the EXIT curve with Gray mapping is presented in the figure. It is found that the Gray mapping has a completely flat EXIT curve, while anti-Gray mapping has a sharper decay. With $l_{\text {map }}=m$, since mapping rule does not change the constellation constraint capacity (CCC), the area under the EXIT curves has to stay almost the same so far as the same modulation is used ( $m=$ constant). With EM, the left most point is further decreased, and the right most point increased. However, the area under the curve is smaller than without EM, because even with $l_{m a p}>m$, still the spectrum efficiency of the modulation itself stays the same $(=m)$.

With the Gaussian assumption for the LLR distribution, the EXIT function of the repetition code is given by

$$
I_{e, v}=J\left(\sqrt{\left.\left(d_{v}-1\right) \cdot J^{-1}\left(I_{a, v}\right)^{2}\right)}\right) .
$$

where $I_{a, v}$ is the variable node input a priori MI and $I_{e, v}$ is its output extrinsic MI. $J()$ and $J^{-1}()$ are the functions that convert the square-root variance of LLR to its corresponding MI, and its inverse, respectively [2]. Obviously, Eq. (5) is corresponding to Eq. (3) for LLR update, with which $I_{a, v}=I_{e, d e m}$ with $I_{e, d e m}$ being the demapper output extrinsic MI.

The check node EXIT function can be approximated by [14]

$$
I_{e, c n d}=1-J\left(\sqrt{d_{c}-1} \cdot J^{-1}\left(1-I_{a, c n d}\right)\right)
$$




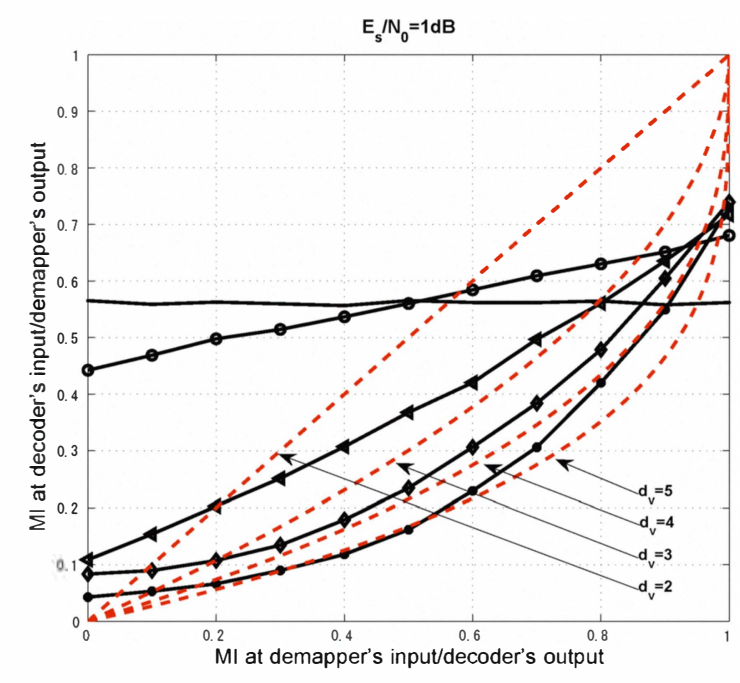

Fig. 4. EXIT chart: 4-QAM extended mapping $l_{m a p}=2 \ldots 5$ combined with repetition code $d_{v}=2 \ldots 5$ without single parity check code.

where

$$
I_{a, c n d}=J\left(\sqrt{d_{v} \cdot J^{-1}\left(I_{a, d e c}\right)^{2}}\right) .
$$

The EXIT function of the whole decoder comprised of the variable and check node decoders can be calculated by combining Eq. (6) and (7) [4], as

$$
I_{e, d e c}=J\left(\sqrt{\left(d_{v}-1\right) \cdot J^{-1}\left(I_{a, d e c}\right)^{2}+J^{-1}\left(I_{e, c n d}\right)^{2}}\right),
$$

with $I_{a, d e c}=I_{e, d e m}$. Since our proposed code use irregular structure of the repetition code, its EXIT function depends on the degree distribution $a_{j}$ of the variable nodes. Our previous publication showed that it is given by the EXIT function of the whole decoder with the proposed structure can be obtained by weighting the segment-wise EXIT functions, as given by Eq. (9), with $a_{j}$ corresponding to their distributions, as

$$
I_{e, d e c}=J\left(\sqrt{\left(d_{v}-1\right) \cdot J^{-1}\left(I_{a, d e c}\right)^{2}+J^{-1}\left(I_{e, c n d}\right)^{2}}\right),
$$

Hence the shape of the combined code EXIT function can be flexibly controlled so that better matching between demapper and decoder curves can be achieved.

Since BICM-ID is a serially concatenated system, its power efficiency and convergence property can be evaluated by the area theorem. It states that when the two EXIT curves are closely enough matched, while the convergence tunnel opens until a MI point close enough to 1.0 , clear threshold in BER performance curve can be achieved, and the distance in SNR from the Shannon limit corresponds to the gap area between the two EXIT curves. In our previous publications [8] [9], however, the degree distributions were obtained by empirically, and no systematic technique was presented. In the next Section, it is shown that determining the optimal degree distribution falls into a convex optimization, for which it can be solved via a linear programming technique.

\section{OPTIMAL DEGREE ALLOCATION}

The code design optimality criterion can be, given $l_{\text {map }}$, written as

Find $a_{i}$ for each $d_{v, i}$

Such that Rate $=\left(d_{c}-1\right) /\left(d_{c} a_{i} \sum \cdot d_{v, i}\right) \rightarrow$ Maximized

Subject to $\sum_{j} a_{j}=1$

$I_{e, d e c}>I_{a, d e c}=I_{e, d e m}$

Now, assume that the check node degree $d_{c}$ is a fixed constant. Then, the optimality criterion of Eq. (10) is equivalent to

Find $a_{i}$ for each $d_{v, i}$

$$
\begin{aligned}
& \text { Such that } \sum_{\text {Subject to }} a_{i} \cdot d_{v, i} a_{j}=1 \text { Minimized } \\
& \qquad I_{e, d e c}>I_{a, d e c}=I_{e, d e m}
\end{aligned}
$$

Now, we introduce an index $w$ representing the MI point and also another parameter $\epsilon_{w}$ representing the acceptable gap between $I_{e, d e c}$ and $I_{a, d e c}$, such that

$$
\begin{array}{r}
I_{e, d e c, w}-I_{a, d e c, w} \geq \epsilon_{w}>0 \\
\text { for } 1 \leq w \leq N,
\end{array}
$$

where $N$ is the number of the indexes. With the parameters and indexes defined above, we can now re-write the optimality criterion, as

Minimize $\sum_{j} a_{j} d_{v, j}$

Subject to

$$
\begin{aligned}
& \sum_{j} a_{j} \cdot d_{v, j} \cdot\left(-J\left(\sqrt{\left(d_{v, j}-1\right) \cdot J^{-1}\left(I_{e, d e m, w}\right)^{2}+J^{-1}\left(I_{e, c n d, w}\right)}\right.\right. \\
& \left.\left.+I_{a, d e m, i}+\epsilon_{i}\right)\right)<0(\text { for } 1 \leq w \leq N) \\
& \sum_{j} a_{j}=1
\end{aligned}
$$

An illustrative diagram of this concept is shown in Fig. 5 where $1 \leq w \leq 11$. Now, given the fact the optimization parameter in Eq. (13) is only $a_{j}$ and the other terms are fixed constant. Furthermore, the index function and constraints are both convex in terms of the optimization variable $a_{j}$. Therefore, the problem falls into a very simple convex optimization, solvable by using a linear programming technique.

\section{NUMERICAL RESULTS}

Table I and II show, as an example, the degree allocation optimization for $l_{m a p}=5, d_{c}=21$ and $\mathrm{SNR}=0.9 \mathrm{~dB}$, where the simplex algorithm was used as a linear programming tool. Table III show the initial values of epsilon for the optimization and Table II show the results. Fig. 6 (a) shows the entire 


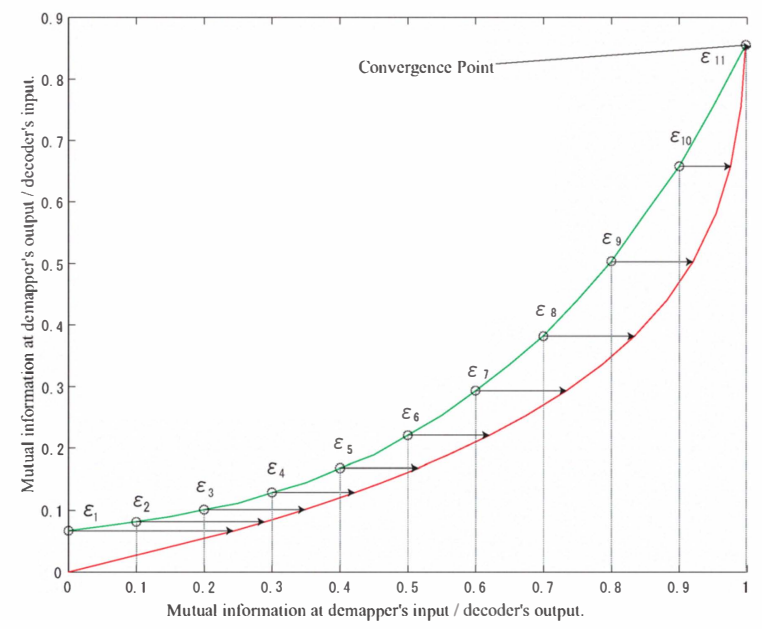

Fig. 5. Schematic Diagram of the Proposed Optimization Technique

portion of the EXIT curves for the de-mapper, decoder with and without optimal degree allocation are shown (In the case without degree optimization, we used the empirically obtained distribution shown in [8], [9]. The two decoder curves are very close each other, but this is due mainly to the coincidence. Fig. 6 (b) and (c) also shows the middle and ending point of the EXIT chart, in the range of $0.385 \leq I_{\text {dem,input }} \leq 0.42$, and $0.98 \leq I_{\text {dem,input }} \leq 1.0$, respectively. It is found that the proposed optimization technique using linear programming result achieves smaller gap between the de-mapper and decoder curves.

TABLE I

DEGREE DISTRIBUTIONS BEFORE OPTIMIZATION

\begin{tabular}{|c||c|c|c|c|c|c|c|c|c|c|}
\hline$d_{v}$ & 1 & 2 & 3 & 4 & 5 & 6 & 7 & 8 & 9 & 10 \\
\hline$a$ & $\frac{1}{10}$ & $\frac{1}{10}$ & $\frac{1}{10}$ & $\frac{1}{10}$ & $\frac{1}{10}$ & $\frac{1}{10}$ & $\frac{1}{10}$ & $\frac{1}{10}$ & $\frac{1}{10}$ & $\frac{1}{10}$ \\
\hline
\end{tabular}

TABLE II

DEGREE DISTRIBUTIONS OBTAINED BY THE OPTIMIZATION

\begin{tabular}{|c||c|c|c|c|c|c|c|c|c|c|}
\hline$d_{v}$ & 1 & 2 & 3 & 4 & 5 & 6 & 7 & 8 & 9 & 10 \\
\hline$a$ & 0 & 0 & 0 & 0 & 0.993 & 0.004 & 0 & 0 & 0 & 0.003 \\
\hline
\end{tabular}

TABLE III

epsilon SETTINGS.

\begin{tabular}{|c||c|c|c|c|c|c|c|c|c|c|}
\hline$w$ & 1 & 2 & 3 & 4 & 5 & 6 & 7 & 8 & 9 & 10 \\
\hline$\epsilon_{w}$ & 0 & 0 & 0 & 0 & 0 & 0.001 & 0.001 & 0.001 & 0.001 & 0.001 \\
\hline \hline$w$ & 11 & 12 & 13 & 14 & 15 & 16 & 17 & 18 & 19 & 20 \\
\cline { 1 - 3 }$\epsilon_{w}$ & 0 & 0 & 0 & 0 & 0 & 0 & 0 & 0 & 0 & 0 \\
\cline { 1 - 8 } &
\end{tabular}

Fig. 7 shows non-optimized and optimized bit error rate (BER) versus $E_{s} / N_{0}$ curves. It is found that when the degree distribution is optimized at $E_{s} / N_{0}=0.9$, the spectrum efficiency is 0.95 bits/symbol, which is 0.05 bits/symol higher the the empirically designed case [8] [9]. The $0.9 \mathrm{~dB}$ threashold for $0.95 \mathrm{bits} / \mathrm{symbol}$ is $1.3 \mathrm{~dB}$ away from the Shannon

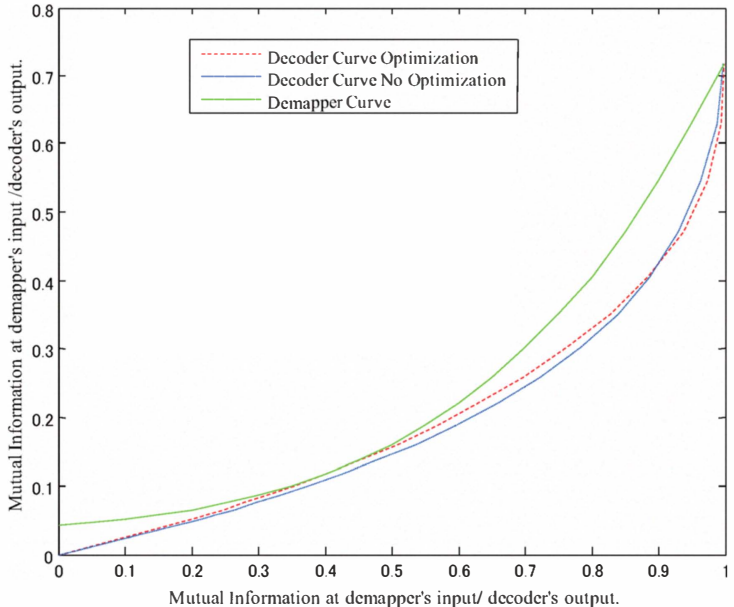

(a) EXIT curves of de-mapper, decoder with and without degree optimization

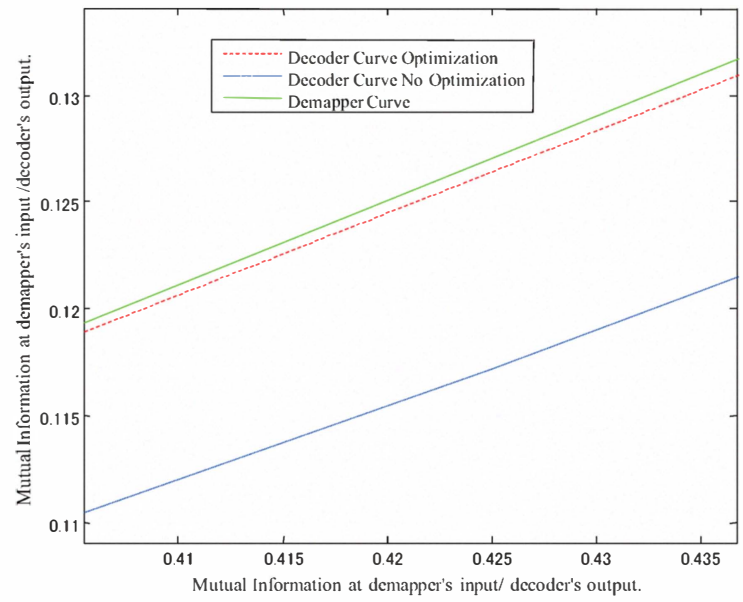

(b) EXIT chart, in the range of $0.385 \leq I_{\text {dem,input }} \leq$ 0.42

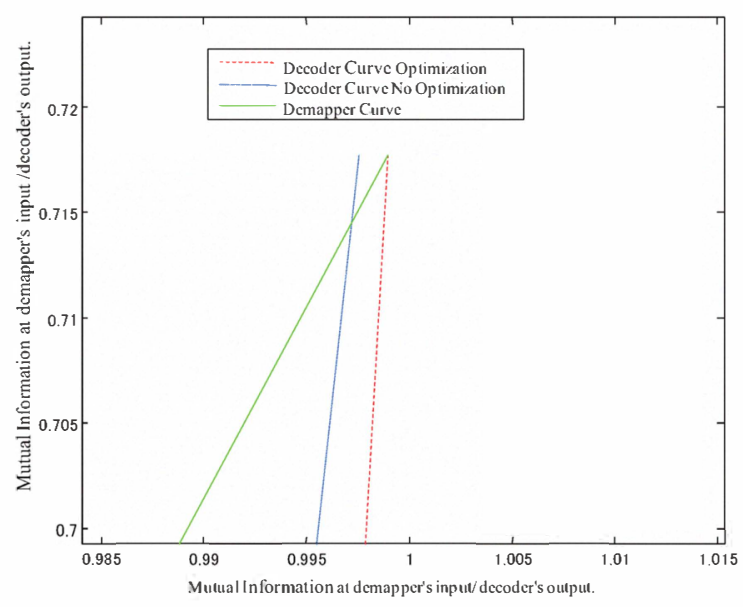

(c) EXIT chart, in the range of $0.98 \leq I_{\text {dem,input }} \leq 1.0$

Fig. 6. EXIT Curves Comparison 




Fig. 7. BER versus $E_{s} / N_{0}$.

limit. Furthermore, it is found that with the degree allocation obtained by using the proposed technique can achieve lower BER floor than the distribution obatined empirically.

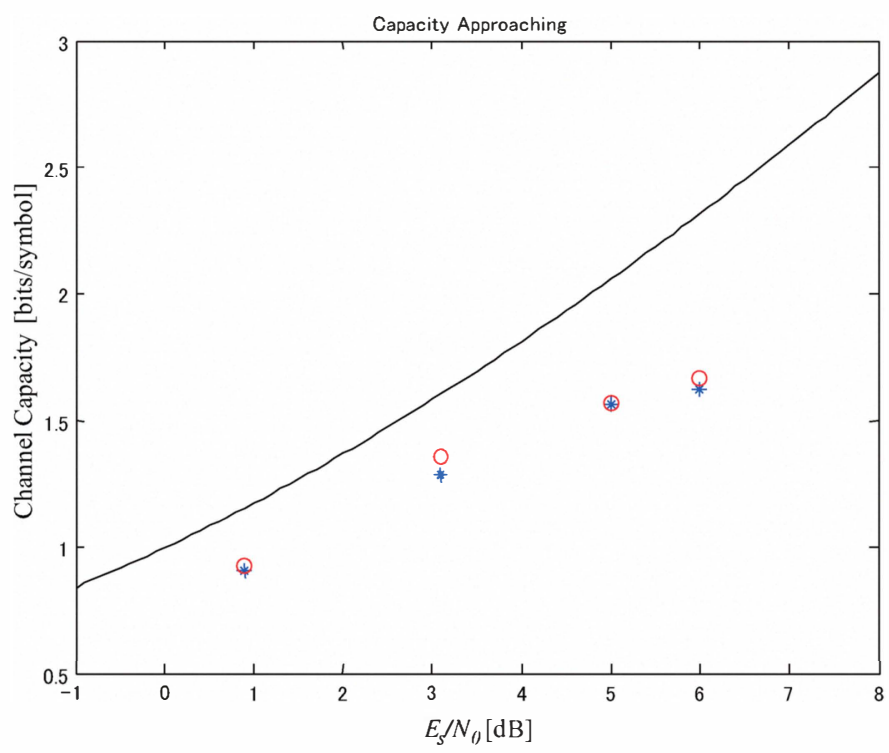

Fig. 8. Threshold $E_{s} / N_{0}$ versus spectrum efficiencies of the codes obtained with and without using the proposed optimization technique

Fig. 8 Threshold $E_{s} / N_{0}$ versus spectrum efficiencies of the codes obtained with and without using the proposed optimization technique

\section{CONCLUSION}

In this paper, we have proposed a new technique for optimal degree distribution optimization, applicable to our proposed very simple BICM-ID technique, irregular repetition and single parity check coded BICM-ID with extended mapping. Previously, the degree distribution for the each variable node was determined empirically by try and error. In this paper, it has been shown that the problem of determining the optimal degree distribution falls into a very simple convex optimization problem, solvable by using a linear programming technique. Results shows we can achieve better matching between the demapper and decoder EXIT curves, by which we can achiever even closer threshold to the Shannon limit and also lower error floor. Finally, it should be emphasized on the same regards as in our previous publications [8] [9] that the complexity for the proposed technique is extremely low, because EM does not require higher order modulation format and no iterations are needed within the decoder itself. Furthermore, quite recently, We have found that by further concatenating the proposed technique with a memory 1 rate 1 code (accumulator), we can completely eliminate the error floor observed in the BER curves shown in this paper. The results has been submitted as another publication [15].

\section{REFERENCES}

[1] G.Caire, G.Taricco, and E.Biglieri, "Bit-interleaved coded modulation," IEEE Trans. on Inform. Theory, vol. 44, no. 3, pp. 927-946, May. 1998.

[2] S. ten Brink., "Convergence behavior of iteratively decoded parallel concatenated codes," IEEE Trans. on Comm., vol. 49, pp. 1727-1737, Oct. 2001.

[3] J. Hagenauer, "The EXIT chart - introduction to extrinsic information transfer in iterative processing," in 12th European Signal Processing Conference (EUSIPCO), 2004, pp. 1541-1548.

[4] S. ten Brink, G.Kramer, and A.Ashikmin, "Design of low-density paritycheck codes for modulation and detection," IEEE Trans. Comm., vol. 52, pp. 670-678, Jun. 2004.

[5] F. Schreckenbach, J. N. Görtz, Hagenauer, and G. Bauch, "Optimized symbol mappings for bit-interleaved coded modulation with iterative decoding," in IEEE GLOBECOM'03, vol. 6, Dec. 2003, pp. 3316-3320.

[6] P. Henkel, "Extended mappings for bit-interleaved coded modulation," in Personal, Indoor and Mobile Radio Communications, 2006 IEEE 17th International Symposium, 2006.

[7] P.Henkel, "Doping of extended mapping for signal shaping," in Vehicular Technology Conference, VTC2007-Spring. IEEE 65th, 2007.

[8] D.Zhao, A.Dauch, and T.Matsumoto, "Bicm-id using extended mapping and repetition code with irregular node degree allocation," in IEEE Vehicular Technology Conference, Barcelona, Apr. 2009, pp. 152-155.

[9] D.Zhao, A.Dauch, and T.Matsumoto, "Modulation doping for repetition coded bicm-id with irregular degree allocation," in ITG/IEEE Workshop on Smart Antennas, Berline, Feb. 2009.

[10] M.Tuchler, R.Koetter, and A.Singer, "Turbo equalization: principles and new results," IEEE Trans. Comm., vol. 50, pp. 754-767, 2002.

[11] L.Hanzo, T.H.Liew, and B.L.Yeap, Turbo Coding, Turbo Equalisaiton and Space-Time Coding for Transmission over Fading Channels. Wiley, 2002.

[12] S. Brink, "Convergence of iterative decoding," Electronics Letters, vol. 35, pp. 806-808, May. 1999.

[13] J. Hagenauer, E. Offer, and L. Papke, "Iterative decoding of binary block and convolutional codes," IEEE Trans. on Inform. Theory, vol. 42, pp. 429-445, Mar. 1996.

[14] S.Liu and D.J.Costello, Error Control Coding (2nd Edition). Prentice Hall, Jun. 2004.

[15] K.Anwar, D. Zhao, and T.Matsumoto, "Simple irregular repeataccumulate codes for bicm-id with extended mapping," in submitted.

[16] X.Wang and H.V.Poor, "Iterative (turbo) soft interference cancellation and decoding for coded cdma," IEEE Trans. on Comm., vol. 47, pp. 1046-1061, 1999.

[17] T.Abe and T.Matsumoto, "Space-time turbo equalization in frequencyselective mimo channels," IEEE Trans. Veh. Tech., pp. 469-475, 2003. 